\title{
The Influence of Iron Ions on Optical Brighteners and Their Application to Cotton Fabrics
}

\author{
Tihana Dekanić $^{1}{ }^{1}$, Tanja Pušić ${ }^{1}$, Ivo Soljačić ${ }^{1}$, Branka Vojnović $^{2, *}$ and Julija Volmajer Valh ${ }^{3}$ \\ 1 Department of Textile Chemistry and Ecology, Faculty of Textile Technology, University of Zagreb, \\ HR-10000 Zagreb, Croatia; tihana.dekanic@ttf.unizg.hr (T.D.); tanja.pusic@ttf.unizg.hr (T.P.); \\ ivo.soljacic@ttf.unizg.hr (I.S.) \\ 2 Department of Applied Chemistry, Faculty of Textile Technology, University of Zagreb, \\ HR-10000 Zagreb, Croatia \\ 3 Institute of Engineering Materials and Design, Faculty of Mechanical Engineering, University of Maribor, \\ SI-2000 Maribor, Slovenia; julija.volmajer@um.si \\ * Correspondence: branka.vojnovic@ttf.unizg.hr
}

\section{check for}

updates

Citation: Dekanić, T.; Pušić, T.; Soljačić, I.; Vojnović, B.; Valh, J.V. The Influence of Iron Ions on Optical Brighteners and Their Application to Cotton Fabrics. Materials 2021, 14 4995. https://doi.org/10.3390/ ma14174995

Received: 7 July 2021

Accepted: 29 August 2021

Published: 1 September 2021

Publisher's Note: MDPI stays neutral with regard to jurisdictional claims in published maps and institutional affiliations.

Copyright: (c) 2021 by the authors. Licensee MDPI, Basel, Switzerland. This article is an open access article distributed under the terms and conditions of the Creative Commons Attribution (CC BY) license (https:/ / creativecommons.org/licenses/by/ $4.0 /)$.

\begin{abstract}
The influence of iron ions at concentrations of $0.2,0.5$, and $1.0 \mathrm{~g} / \mathrm{L}$ on optical brighteners of the groups stilbene and biphenyl in solution and on cotton fabric was investigated. Both groups of optical brighteners are intended for detergent formulations. The influence of iron ions was studied by absorption and fluorescence spectra in solution and by whiteness degree, identifying color differences using CIEL $\mathrm{a}^{*} \mathrm{~b}^{*}$ coordinates and Ultraviolet Protection Factor (UPF) of cotton fabrics. The obtained results in solutions and cotton fabrics showed different behavior of optical brighteners stilbene and biphenyl in the presence of iron. Stilbene compounds with metal ions produced new species capable of absorbing in the UV-B region of the spectrum. A biphenyl compound in combination with iron had no effect on the absorption properties. Both optical brighteners were influenced by iron ions in the sense of fluorescence quenching. The influence of iron ions in single- and two-bath treatments of cotton fabrics after one cycle on whiteness degree and UPF was negligible.
\end{abstract}

Keywords: optical brighteners; solutions; iron ions; absorption; fluorescence; cotton fabric; whiteness degree; Ultraviolet Protection Factor (UPF)

\section{Introduction}

Optical brighteners (OBs) are special compounds that contain a fluorescent system instead of a chromophore and are intended for optical and UV protection applications [1]. The basic requirement for these compounds is the ability to absorb electromagnetic radiation (EMR) in the UV spectrum. It is usually associated with the molecules with planar configuration (e.g., metal complexes), conjugated double bonds, or high resonance stability, outstanding among them are aromatic compounds with an electron donor group (e.g., $-\mathrm{NH}_{2}$ ) or acidic protons (phenols and anilines) [2]. Optical brighteners absorb maximally in the near UV, from 340 to $380 \mathrm{~nm}$, and emit in the visible part of the spectrum at 425 to $450 \mathrm{~nm}$ [3]. From a chemical point of view, OBs can be derivatives of stilbene, coumarin, benzoxazole, benzo[b]furan, etc. [4,5].

Cotton, an important biopolymer in the manufacture of woven and knitted fabrics, cannot provide adequate protection against UV radiation. UV protection of cotton textiles can be improved by fabric weaving construction parameters and finishes [6-14]. OBs are known finishing additives that can improve the UV protection of cotton textiles [15-17]. Washing such treated cotton materials with detergents without OBs leads to a loss of optical and UV protection properties. According to previous studies, UPF reaches the level of untreated fabric after 10 washing cycles $[17,18]$. The role of OBs in detergent formulation for washing cotton fabrics is to improve the optical and UV properties. Previous research has mainly focused on the optical properties of OBs in detergents but not on the UV 
properties [3,19-21]. OBs in detergents can provide UV protection properties as an added value of textiles in the washing process [18,22-26]. Stilbene and biphenyl derivatives are most commonly used as detergent OBs $[3,19,20]$. Such washing effects can be blocked by the presence of chlorine and various types of metal ions, depending on their concentration and composition. They can reduce the absorption and/or quench fluorescence and reduce whiteness [3,27-30]. The reason Fe ions reduce the fluorescence intensity of the $\mathrm{OB}$ is most likely due to impact of the Fe ion on the electronically excited molecule followed by a reverse electron-transfer reaction, which leads to the system returning to its original unexcited state. To the best of our knowledge, the effect of detergent with OBs in the presence of metal ions on UV protection properties has not been previously researched.

The importance of UV protection of cotton materials when washed with the detergents containing OBs was the motivation for the present study. There are many studies in which UV protection of cotton materials has been improved by fabric weaving construction parameters and finishes. OBs in detergents can also increase the UV protection properties as an added value of textiles in the washing process. In this study, the basis was to research the ability of the UV protection of the cotton materials when washed with the detergent containing optical brighteners (OBs) and in the presence of fluorescence quencher. For that purpose, in a first step, aqueous solutions of stilbene and biphenyl OBs were analyzed under the influence of iron ions using absorption and fluorescence spectra. The application of OBs in the presence and absence of iron ions was carried out on standard cotton fabrics by single- and two-bath treatments. The optical and protective properties of cotton fabrics before and after treatments were characterized by whiteness $\left(\mathrm{W}_{\mathrm{CIE}}\right)$, spectral parameters $\left(a^{*}\right.$ and $\left.b^{*}\right)$, and by Ultraviolet Protection Factor (UPF).

\section{Materials and Methods}

\subsection{Materials}

The chemical structures of the OBs with stilbene (S) and biphenyl structures (B) structures are shown in Table 1.

Table 1. Chemical structure of the fluorescent compounds.

$\begin{array}{ccc}\text { Label } & \text { Name } \\ \text { disodium } \\ \text { Weight (g/mol) }\end{array}$

Analytical evaluation of the stilbene and biphenyl OBs in solutions with concentrations of $0.08 \%, 0.12 \%$, and $0.25 \%$ was carried out. Table 2 shows the labeling of OBs with different concentrations.

The iron standard solution $\mathrm{Fe}\left(\mathrm{NO}_{3}\right)_{3}$ in $\mathrm{HNO}_{3} 0.5 \mathrm{~mol} / \mathrm{L} 1000 \mathrm{mg} / \mathrm{L}$ Fe Certipur ${ }^{\circledR}$ from Merck served as the source of iron ions. Appropriate aliquots were taken by diluting the stock solution in distilled water depending on the desired addition of iron ions.

Concentrations of $0.2,0.5$, and $1.0 \mathrm{mg} / \mathrm{L}$ of the iron standard solution were added to the OBs, as shown in Table 3. 
Table 2. Labeling of the optical brighteners solutions.

\begin{tabular}{ccc}
\hline OBs Derivative & $\mathbf{w}_{\mathbf{O B}}(\mathbf{\%})$ & Label \\
\hline \multirow{2}{*}{ Stilbene } & 0.08 & $\mathrm{~S}-0.08$ \\
& 0.12 & $\mathrm{~S}-0.12$ \\
& 0.25 & $\mathrm{~S}-0.25$ \\
\hline \multirow{2}{*}{ Biphenyl } & 0.08 & $\mathrm{~B}-0.08$ \\
& 0.12 & $\mathrm{~B}-0.12$ \\
& 0.25 & $\mathrm{~B}-0.25$ \\
\hline
\end{tabular}

Table 3. Labelling of OBs with iron ions.

\begin{tabular}{ccccc}
\hline & \multicolumn{5}{c}{ Label } \\
\hline \multirow{2}{*}{$\gamma_{\text {Fe }}(\mathbf{m g} / \mathrm{L})$} & $\mathbf{0}$ & $\mathbf{0 . 2}$ & $\mathbf{0 . 5}$ & $\mathbf{1 . 0}$ \\
\hline \multirow{2}{*}{ W $_{\mathrm{S}}(\%)$} & S-0.08 & S-0.08_0.2 & S-0.08_0.5 & S-0.08_1.0 \\
\cline { 2 - 5 } & S-0.12 & S-0.12_0.2 & S-0.12_0.5 & S-0.12_1.0 \\
\cline { 2 - 5 } & S-0.25 & S-0.25_0.2 & S-0.25_0.5 & S-0.25_1.0 \\
\cline { 2 - 5 } W $_{\text {B }(\%)}$ & B-0.08 & B-0.08_0.2 & B-0.08_0.5 & B-0.08_1.0 \\
\cline { 2 - 5 } & B-0.12 & B-0.12_0.2 & B-0.12_0.5 & B-0.12_1.0 \\
\hline & B-0.25 & B-0.25_0.2 & B-0.25_0.5 & B-0.25_1.0
\end{tabular}

The application of OBs was realized by the washing process of a cotton fabric in plain weave with the surface mass of $175.6 \mathrm{~g} / \mathrm{m}^{2}$ and the density in warp/weft direction $25 / 25$ yarns $/ \mathrm{cm}$. An ECE Color fastness Test Detergent 77 (5 g/L) without OBs in its composition was used [31].

\subsection{Treatments}

The treatments of cotton fabrics were carried out in a Linitest apparatus, Original Hanau as follows:

Single-bath treatment-simultaneous washing of cotton fabrics with $0.2 \mathrm{mg} / \mathrm{L}$, $0.5 \mathrm{mg} / \mathrm{L}$, and $1.0 \mathrm{mg} / \mathrm{L}$ iron standard solutions added to ECE reference detergent $(5 \mathrm{~g} / \mathrm{L})$ with stilbene and biphenyl $\mathrm{OBs}(0.08 \%, 0.12 \%$, and $0.25 \%)$ at $60{ }^{\circ} \mathrm{C}$, bath ratio 1:20, duration $30 \mathrm{~min}$.

Two-bath treatment- cotton fabrics were treated with iron ions at concentrations of $0.2,0.5$, and $1.0 \mathrm{mg} / \mathrm{L}$ (at $60^{\circ} \mathrm{C}$, bath ratio 1:20, duration for $30 \mathrm{~min}$ ). Samples were rinsed with water and washed with ECE reference detergent containing stilbene and biphenyl OBs $(0.08 \%, 0.12 \%$, and $0.25 \%)$ at $60{ }^{\circ} \mathrm{C}$, bath ratio $1: 20$, for $30 \mathrm{~min}$.

Treated cotton samples specially marked with asterisks, ${ }^{*}$ for single-bath treatment and ** for two-bath treatment, were dried under ambient conditions and evaluated objectively by methods.

\subsection{Methods}

UV/Vis spectrophotometer Lambda 20, Perkin Elmer, was used under the following measurement conditions: range from 200 to $500 \mathrm{~nm}$, aperture size $2 \mathrm{~nm}$, and reading-off range $1 \mathrm{~nm}$ for absorption spectra of solutions. Fluorimeter F-7000, Hitachi (Tokyo, Japan), was used to measure fluorescence intensity in the wavelength range of 300 to $600 \mathrm{~nm}$, at excitation wavelength of $250 \mathrm{~nm}$, and wavelength scan speed of $240 \mathrm{~nm} / \mathrm{min}$.

The spectral characteristics of cotton fabrics before and after treatments were measured using a Spectraflash SF 600+ CT, Datacolor, remission spectrophotometer in the measurement range of 360 to $700 \mathrm{~nm}$, with an aperture of $20 \mathrm{~mm}$, standard illumination $\mathrm{D} 65$, and reported as the mean of three individual measurements. The $\mathrm{W}_{\mathrm{CIE}}$ was calculated automatically, according to EN ISO 105-J02 [32]. Transmittance was measured with a UV/V 
is spectrophotometer Varian-Cary 50/Solascreen, using a software package to calculate specific UPF values using the in vitro method according to AS/NZS 4399:1996 [33,34]. UPF is an expression indicating the degree of protection against $U V$ radiation and is calculated automatically from the transmittance $T(\lambda)$, according to the following equation:

$$
U P F=\frac{\sum_{\lambda=290}^{400} E(\lambda) \cdot \varepsilon(\lambda) \cdot \Delta \lambda}{\sum_{\lambda=290}^{400} E(\lambda) \cdot T(\lambda) \cdot \varepsilon(\lambda) \cdot \Delta \lambda}
$$

where:

$E(\lambda)$-relative erythemal spectral effectiveness $\left(\mathrm{W} \mathrm{m}^{-2} \mathrm{~nm}^{-1}\right)$;

$\varepsilon(\lambda)$-solar spectral irradiance;

$\Delta \lambda$-measured wavelength interval (nm);

$T(\lambda)$-average spectral transmittance of the specimen (\%).

\section{Results and Discussion}

The effect of iron ions on the optical brighteners in solution was assessed using absorption and fluorescence spectra (Figures 1-4; Tables 4 and 5).

The application of OBs with iron ions on cotton fabrics was analyzed by whiteness degree, CIEL*a* $b^{*}$ coordinates, and UPF (Figures 5-9, Table 6).

\subsection{Absorption and Fluorescence Curves of Optical Brighteners in a Solution}

In Figure 1a,b, the absorbance and fluorescence spectra of stilbene $\mathrm{OB}$ are plotted for three different concentration ranges. The results obtained showed that increased concentration of optical brightener resulted in higher absorption of UV radiation (S-0.08 $<$ S-0.12 $<$ S-0.25), Figure 1a. The stilbene optical brightener exhibits two absorption peaks, one in the UVA (at wavelength $330 \mathrm{~nm}$ ) region and one in the UV-C (at wavelength $273 \mathrm{~nm}$ ) region. The fluorescence maximum of stilbene $\mathrm{OB}$ is at $430 \mathrm{~nm}$ (Figure $1 \mathrm{~b}$ ).

Figure 2 shows the comparison of the absorption and fluorescence spectra of the optical brighteners used on stilbenes as a function of the concentration of iron ions. The absorption spectra of stilbene $\mathrm{OB}$ at three concentrations after addition of ferrous ions show a bathochromic and hypochromic shift. There was a bathochromic shift of the absorption maximum (Figure 2a; Table 4) to higher wavelengths, i.e., to the UV-A (375 nm) and UV-B $(286 \mathrm{~nm})$ regions, which is particularly evident at higher concentrations of optical brighteners. The highest hypochromic shift was registered at the concentration of iron ions $0.5 \mathrm{mg} / \mathrm{L}$ by the absorption maximum (Figure 2a).

The iron ions and the optical brightener stilbene (Figure 2a; Table 4) generated a different species compared to the parent compound (at about $360 \mathrm{~nm}$ ) that absorbs in the UV-A region, just like a OB. These likely new complexes open up a platform for original investigative questions. 


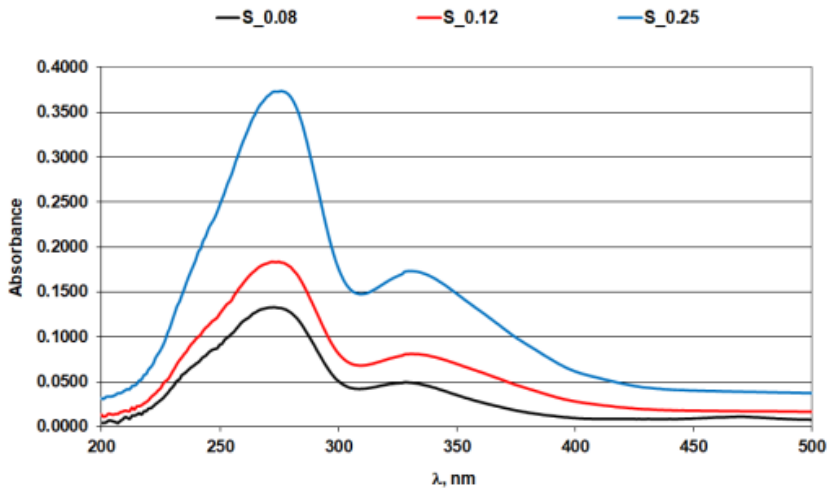

(a)

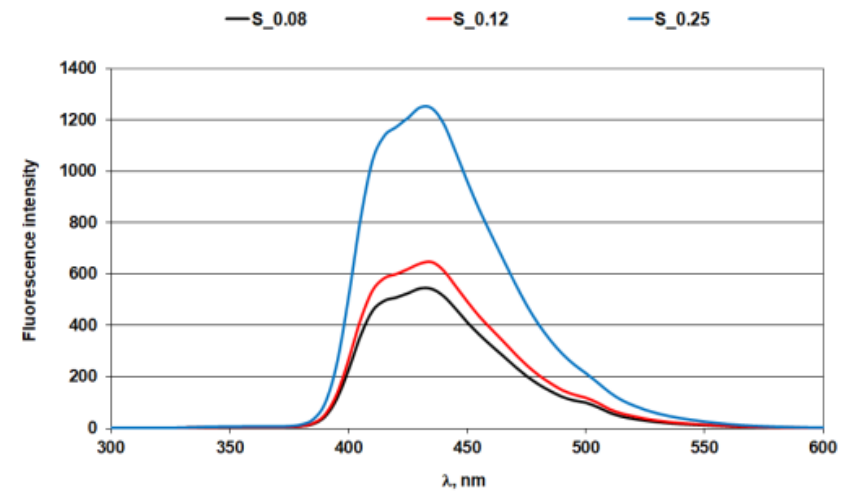

(b)

Figure 1. Stilbene optical brightener: (a) absorption spectra; (b) fluorescence spectra.
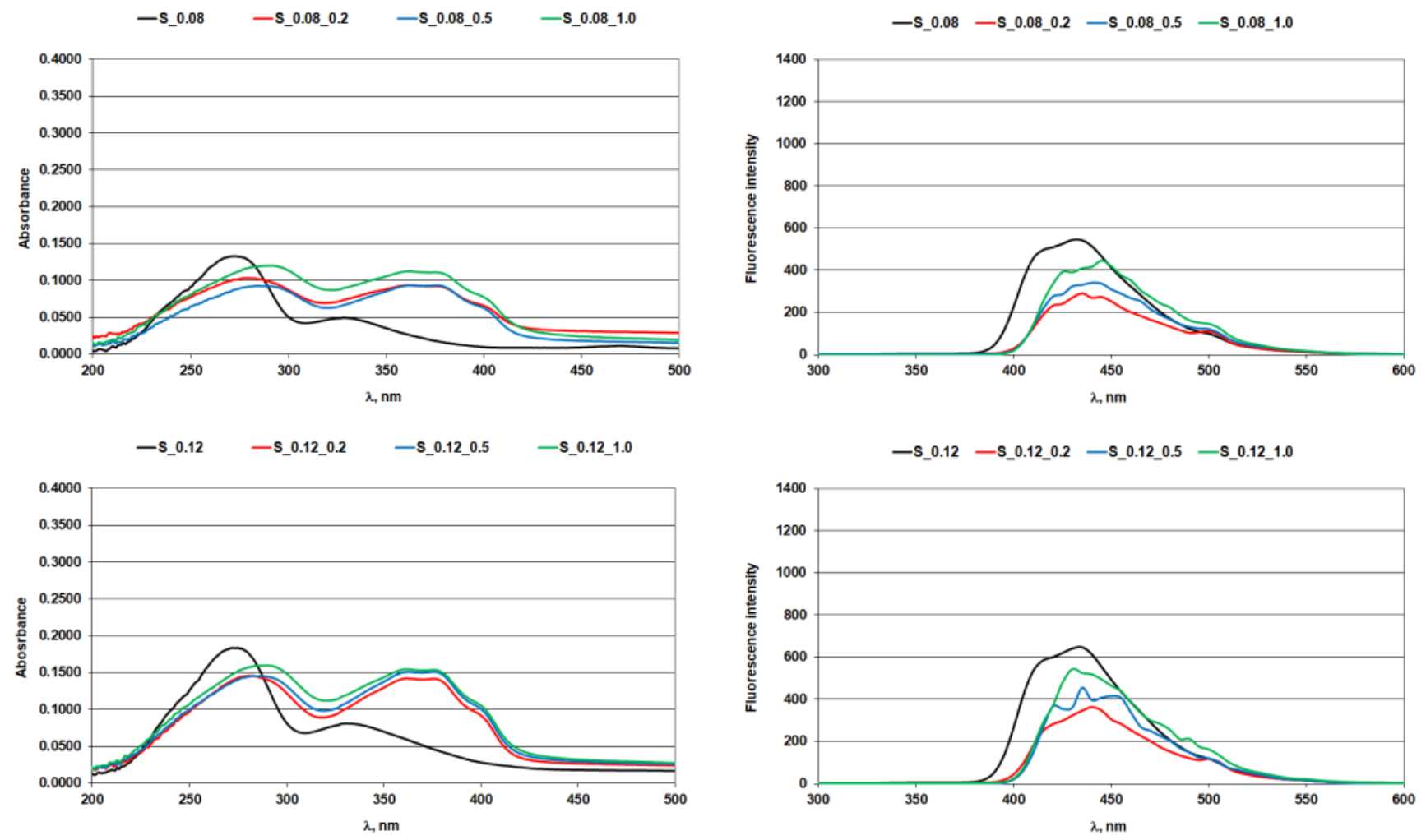

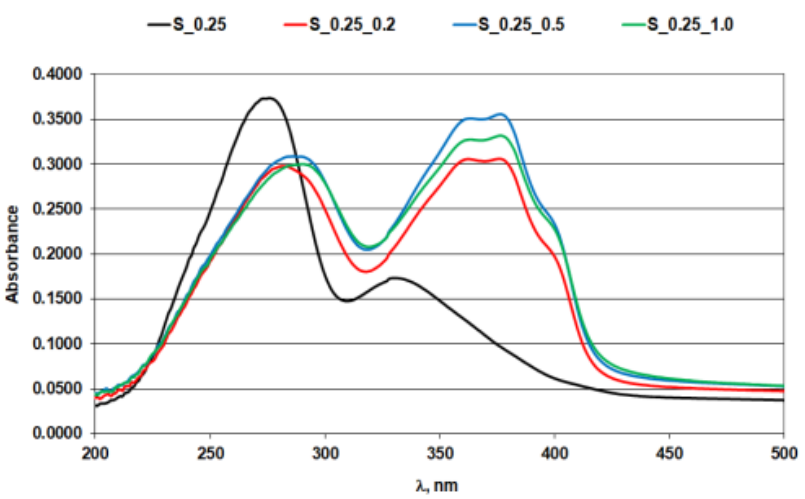

(a)

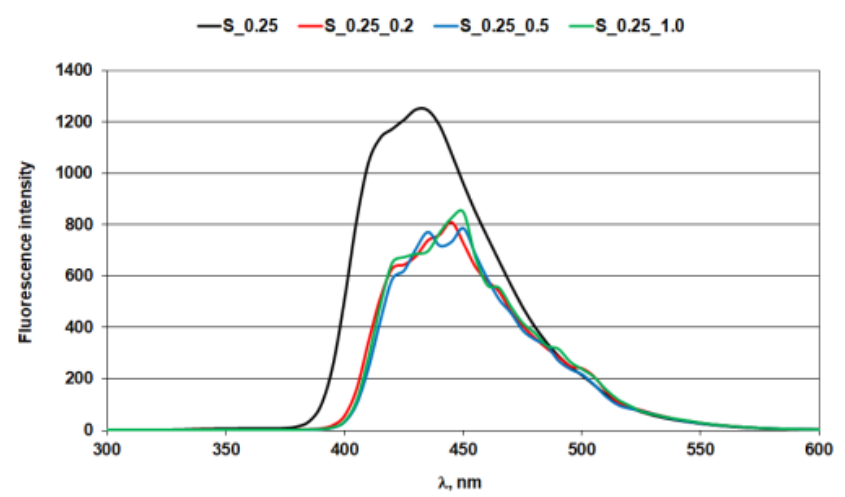

(b)

Figure 2. Stilbene optical brightener with iron ions: (a) absorption spectra; (b) fluorescence spectra. 


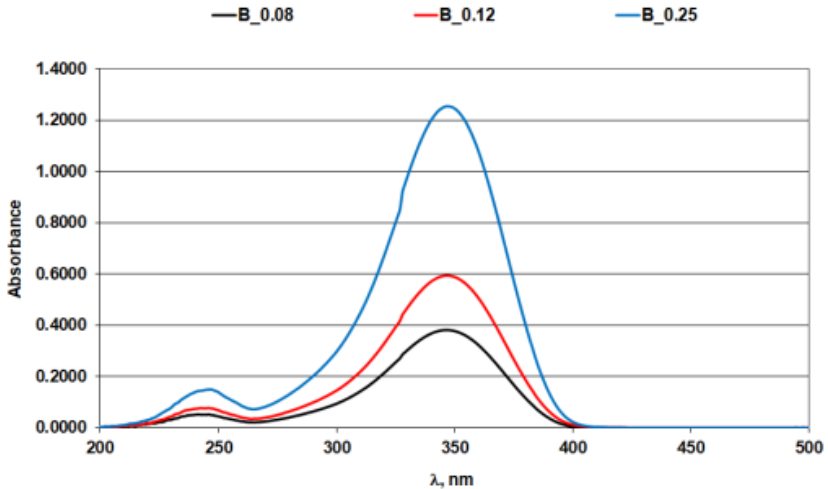

(a)

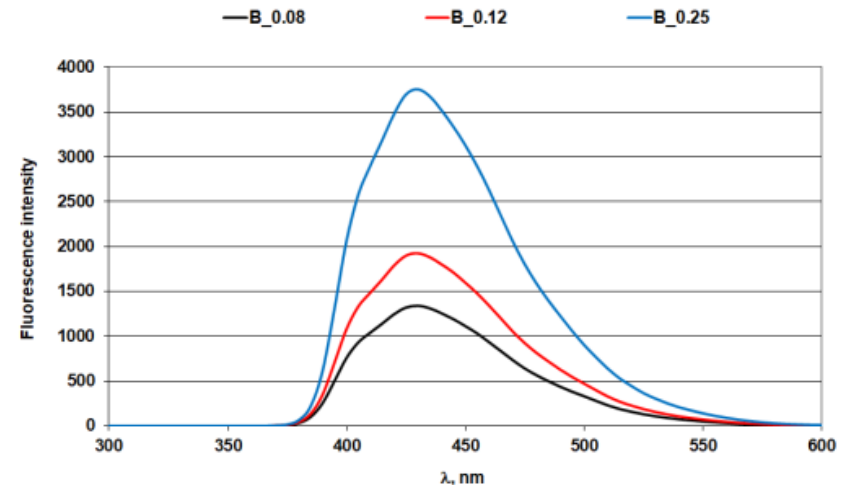

(b)

Figure 3. Biphenyl optical brightener: (a) absorption spectra; (b) fluorescence spectra.
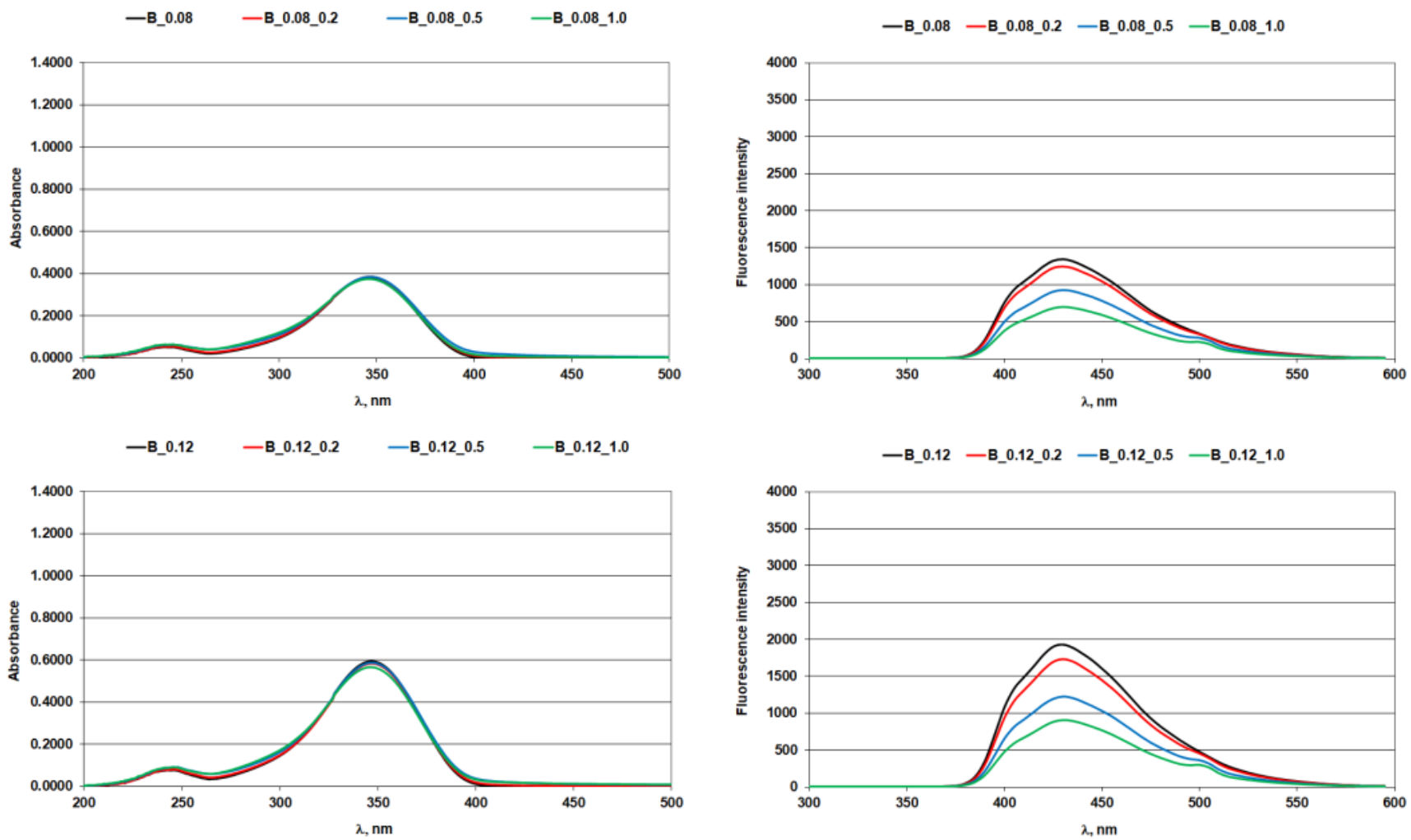

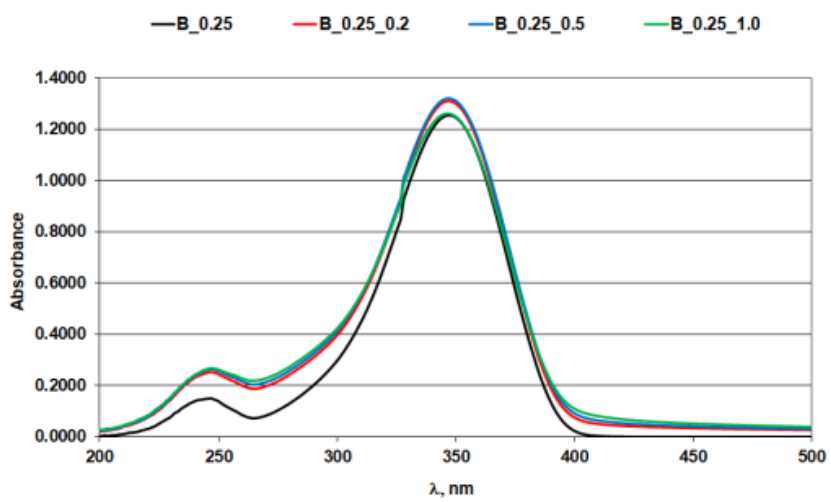

(a)

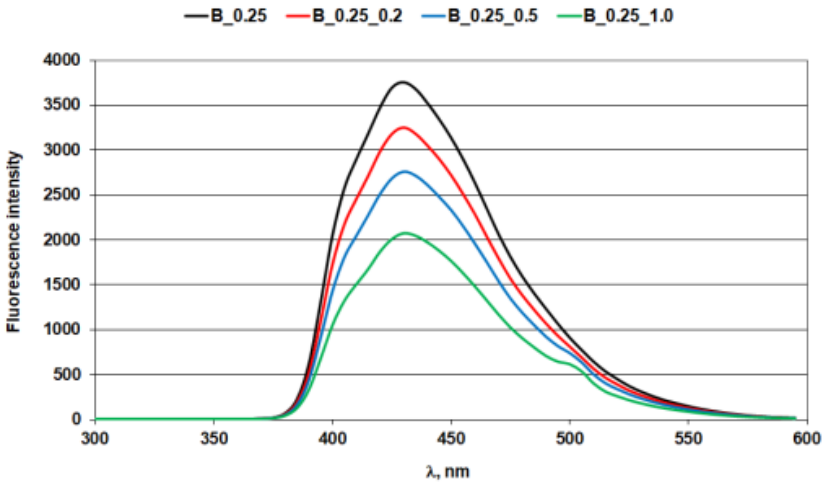

(b)

Figure 4. Biphenyl optical brightener with iron ions: (a) absorption spectra; (b) fluorescence spectra. 
Table 4. Wavelength values at peak absorption of stilbene optical brightener solution with iron ion, as dependent upon the UV region of absorption.

\begin{tabular}{cccc}
\hline \multirow{2}{*}{ Label } & \multicolumn{3}{c}{ Spectrum } \\
\cline { 2 - 4 } & UV-A (315-400) & UV-B (280-315) & UV-C (100-280) \\
\hline S-0.08 & 330 & $\lambda_{\max }$ & 273 \\
\hline S-0.08_0.2 & 375 & - & - \\
\hline S-0.08_0.5 & 375 & 286 & - \\
\hline S-0.08_1.0 & 375 & 286 & - \\
\hline S-0.12 & 330 & - & - \\
\hline S-0.12_0.2 & 375 & 286 & - \\
\hline S-0.12_0.5 & 375 & 286 & 273 \\
\hline S-0.12_1.0 & 375 & 286 & - \\
\hline S-0.25 & 330 & - & - \\
\hline S-0.25_0.2 & 375 & 286 & - \\
\hline S-0.25_0.5 & 375 & 286 & \\
\hline S-0.25_1.0 & 375 & 286 & - \\
\hline
\end{tabular}

Table 5. Wavelength values at peak absorption of biphenyl optical brightener solution with iron ion, as dependent upon the UV region of absorption.

\begin{tabular}{cccc}
\hline \multirow{2}{*}{ Label } & \multicolumn{3}{c}{ Spectrum } \\
\cline { 2 - 4 } & UV-A (315-400) & UV-B (280-315) & UV-C (100-280 nm) \\
\hline B-0.08 & \multicolumn{3}{c}{$\lambda_{\max }$} \\
\hline B-0.08_0.2 & 347 & - & 246 \\
\hline B-0.08_0.5 & 347 & - & 246 \\
\hline B-0.08_1.0 & 347 & - & 246 \\
\hline B-0.12 & 347 & - & 246 \\
\hline B-0.12_0.2 & 347 & - & 246 \\
\hline B-0.12_0.5 & 347 & - & 246 \\
\hline B-0.12_1.0 & 347 & - & 246 \\
\hline B-0.25 & 347 & - & 246 \\
\hline B-0.25_0.2 & 347 & - & 246 \\
\hline B-0.25_0.5 & 347 & - & 246 \\
\hline B-0.25_1.0 & 347 & - & \\
\hline
\end{tabular}




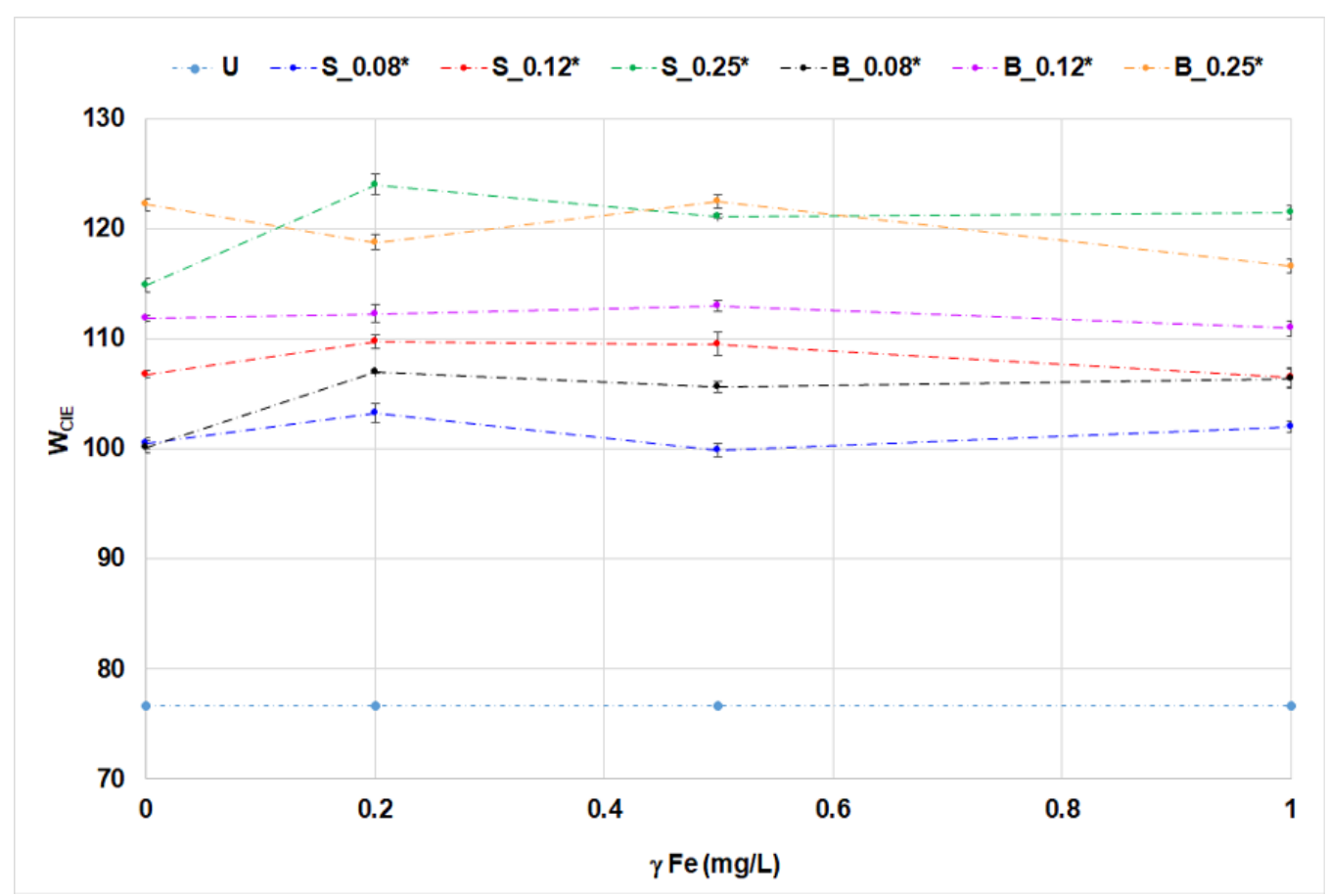

Figure 5. Whiteness degree $\left(\mathrm{W}_{\mathrm{CIE}}\right)$ of cotton samples treated in single-bath treatment.

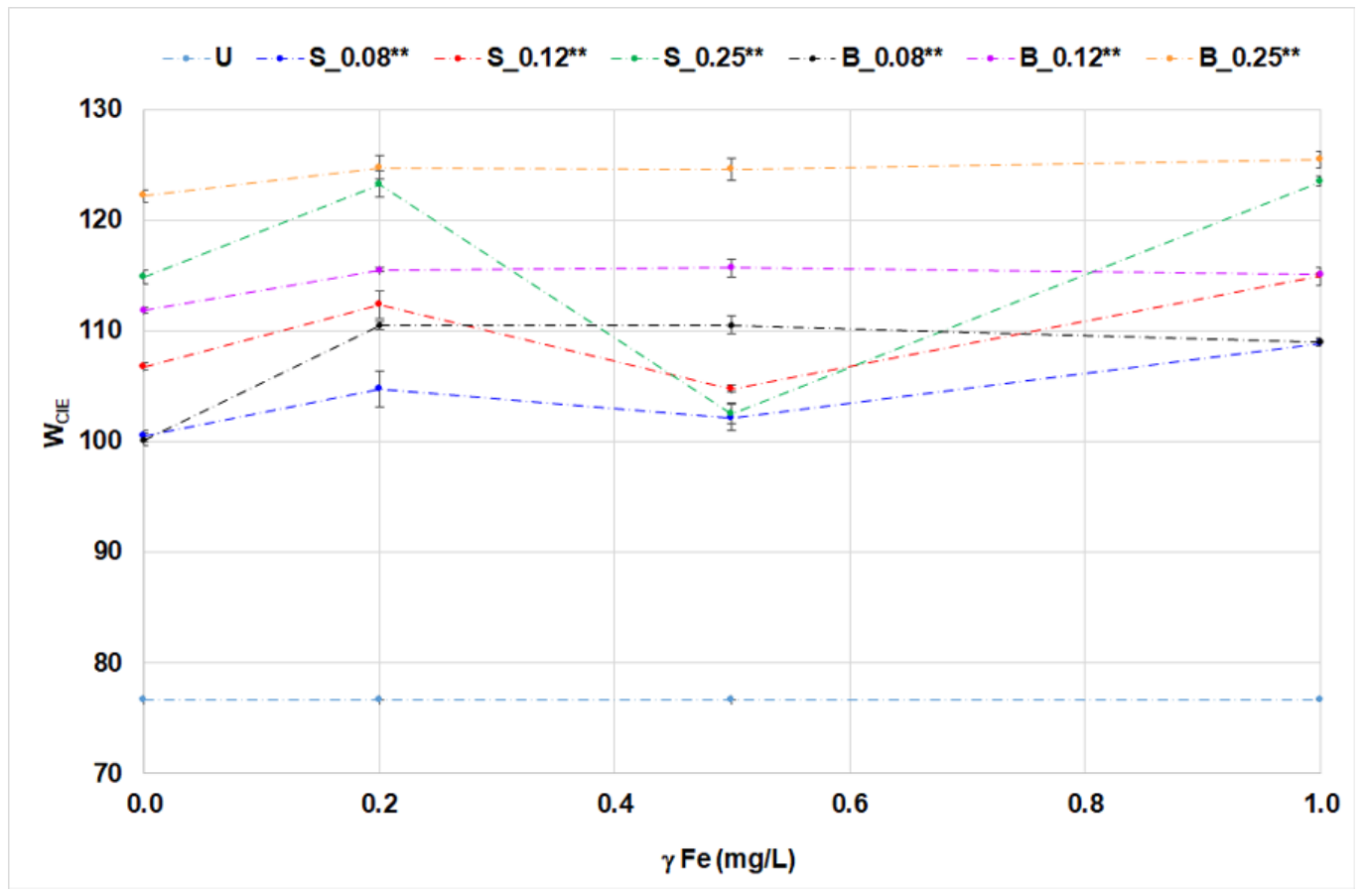

Figure 6. Whiteness degree $\left(\mathrm{W}_{\mathrm{CIE}}\right)$ of cotton samples treated in two-bath treatment. 

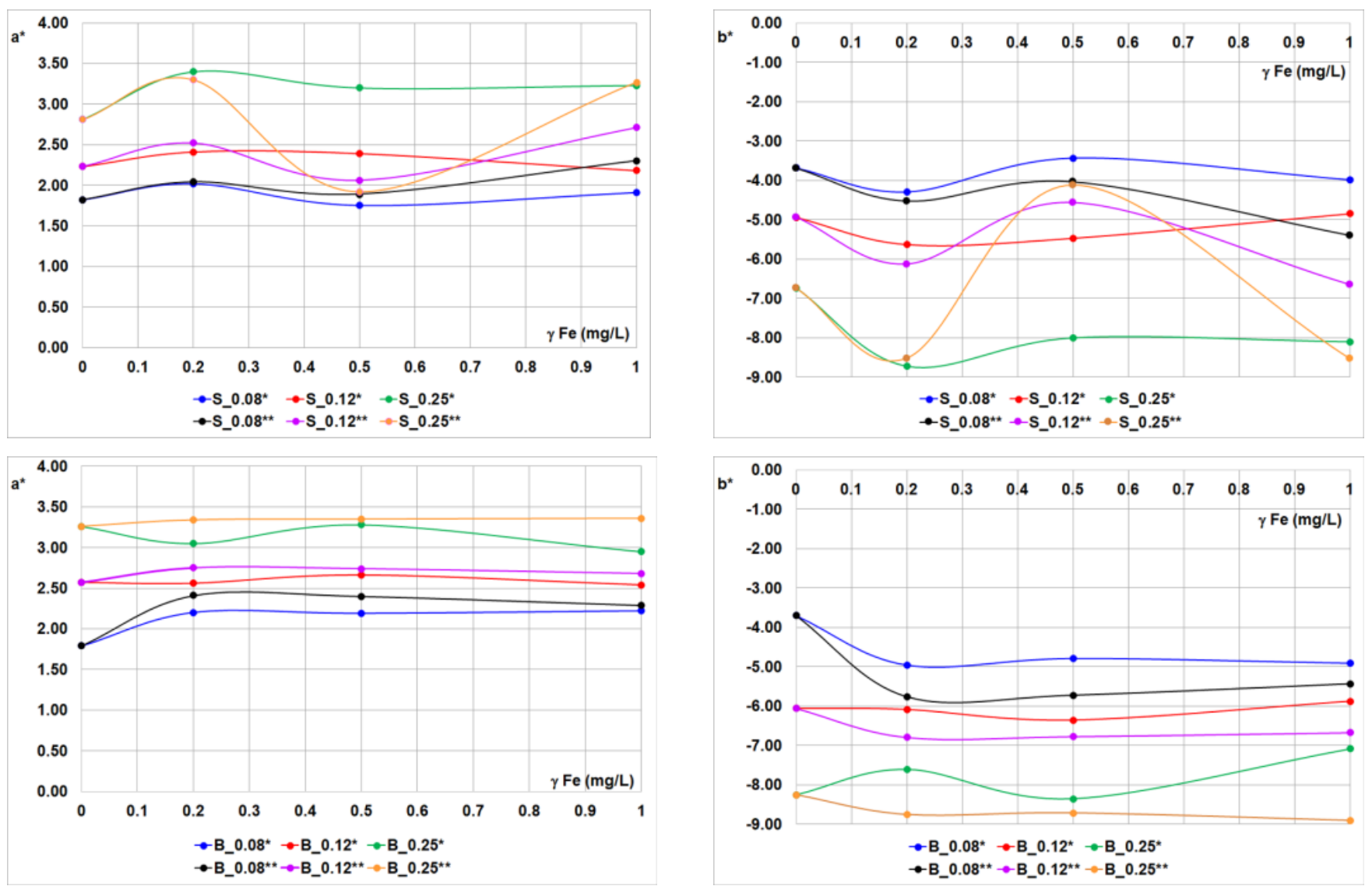

Figure 7. Spectral coordinates, $\mathrm{a}^{*}$ and $\mathrm{b}^{*}$, of cotton fabrics treated in a single-bath and two-bath method.

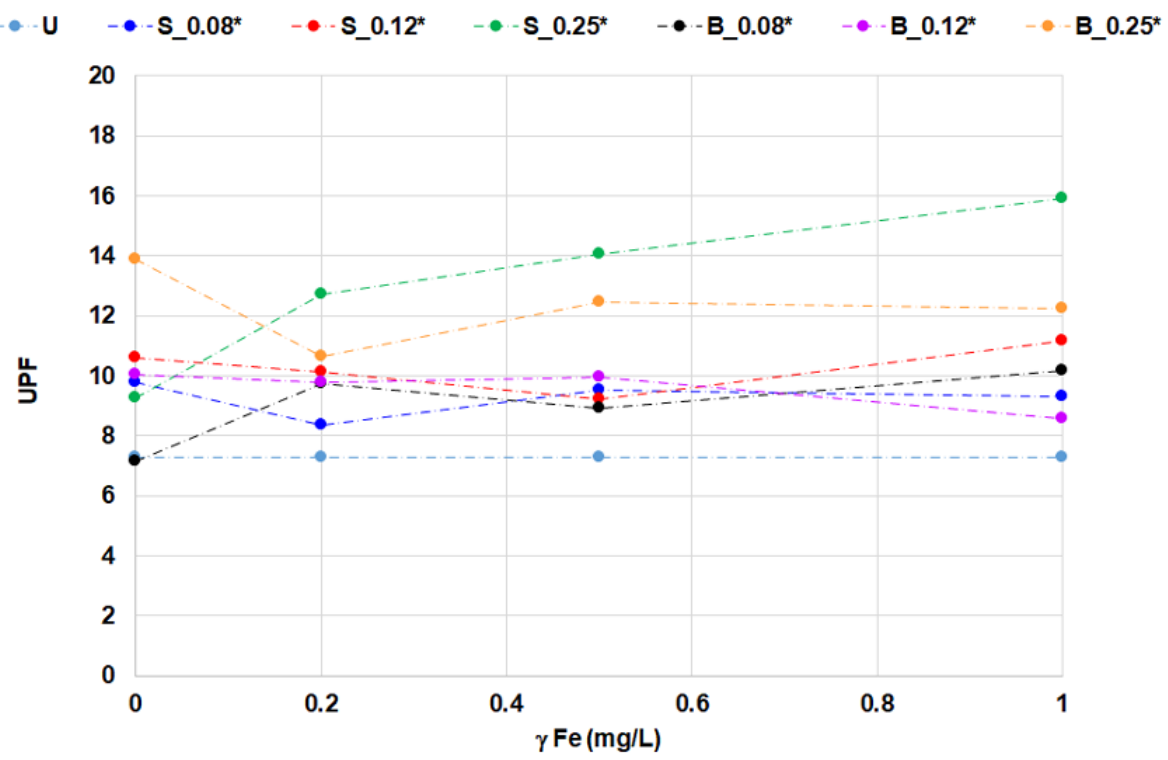

Figure 8. UPF of cotton fabrics treated in single-bath treatment. 


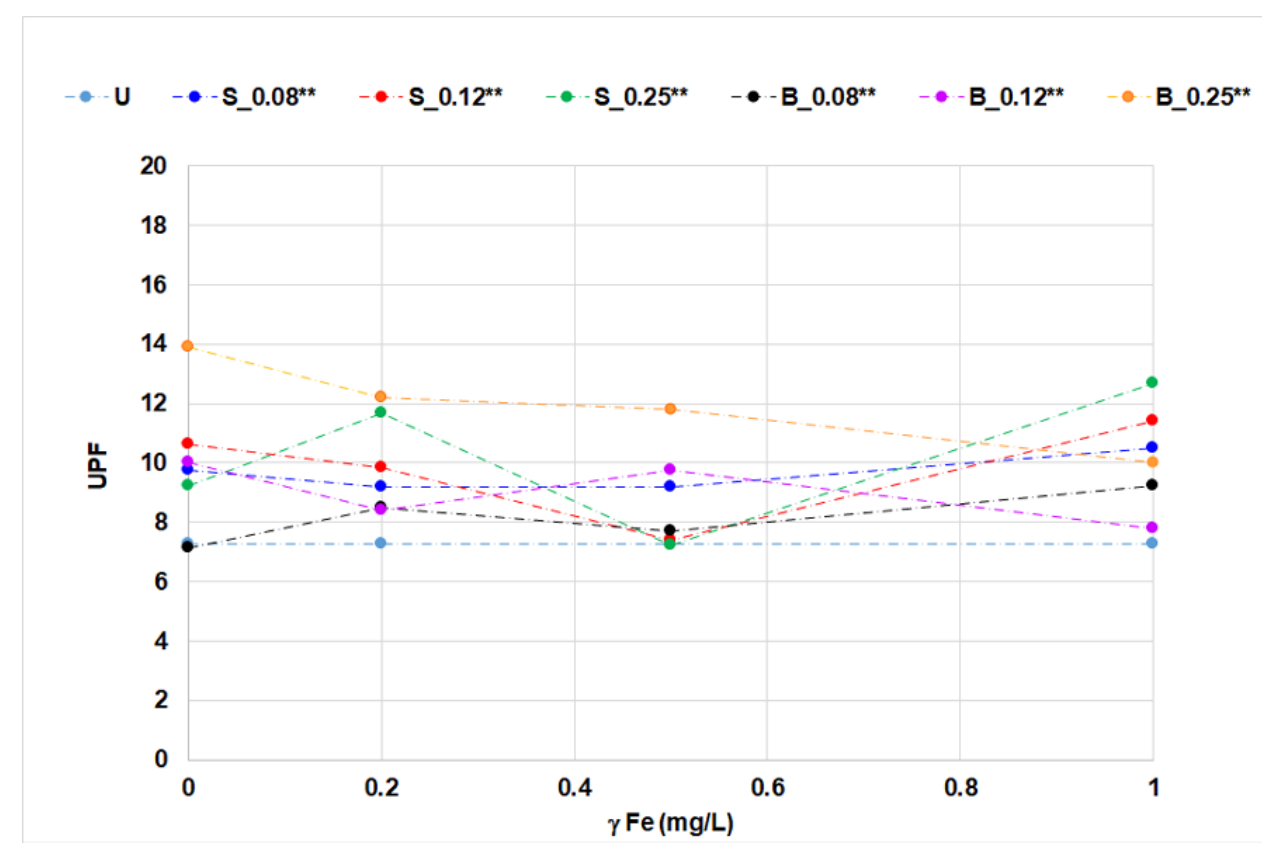

Figure 9. UPF of cotton fabrics treated in two-bath treatments.

Table 6. Lightness of cotton fabric samples treated with iron ions.

\begin{tabular}{|c|c|c|c|c|}
\hline Cotton Fabrics Treatment & \multicolumn{4}{|c|}{$\gamma_{\mathrm{Fe}}(\mathrm{mg} / \mathrm{L})$} \\
\hline Single-bath & 0 & 0.2 & 0.5 & 1.0 \\
\hline S_0.08* & 93.23 & 93.23 & 93.49 & 93.31 \\
\hline S_0.12* & 93.52 & 93.42 & 93.68 & 93.61 \\
\hline S_0.25* & 93.47 & 93.57 & 93.75 & 93.72 \\
\hline B_0.08* & 93.06 & 93.56 & 93.31 & 93.41 \\
\hline B_0.12* & 93.51 & 93.66 & 93.39 & 93.46 \\
\hline B_0.25* & 93.70 & 93.45 & 93.64 & 93.54 \\
\hline Cotton Fabrics Treatment & \multicolumn{4}{|c|}{$\gamma_{\mathrm{Fe}}(\mathrm{mg} / \mathrm{L})$} \\
\hline Two-bath & 0 & 0.2 & 0.5 & 1.0 \\
\hline S_0.08** & 93.23 & 93.42 & 93.28 & 93.53 \\
\hline S_0.12** & 93.52 & 93.61 & 93.35 & 93.70 \\
\hline S_ $0.25^{* *}$ & 93.47 & 93.67 & 93.27 & 93.77 \\
\hline B_0.08** & 93.06 & 93.51 & 93.59 & 93.51 \\
\hline B_0.12** & 93.51 & 93.67 & 93.80 & 93.70 \\
\hline B_ $0.25^{* *}$ & 93.70 & 93.78 & 93.92 & 93.91 \\
\hline Untreated & 93.60 & 93.60 & 93.60 & 93.60 \\
\hline
\end{tabular}

In spite of the fact that the mechanism of fluorescence quenching (or reduction of fluorescence intensity) has not been investigated in this paper, it can be seen from the shape of the fluorescence curve that quenching occurred most probably due to the formation of a complex between iron ions and $\mathrm{OB}$. This newly formed complex had less pronounced fluorescent properties, which is manifested in a decrease in fluorescence intensity (Figure 2b). This effect was more pronounced with lower concentrations of $\mathrm{OB}$, which were still sufficient to form a complex with iron presented in the solution. On the other hand, the shape of the fluorescence curve in Figure $2 \mathrm{~b}$ shows that the lowest concentrations of iron $(0.2 \mathrm{mg} / \mathrm{L})$ with a lower concentration of $\mathrm{OB}(0.12 \%)$ caused the largest decrease in fluorescence intensity, and the shape of the curve 
is identical to the curve of $\mathrm{OB}$ but with lower fluorescence intensity. It is possible that further increasing the concentration of iron, precisely because of its excess, complexes with a different ratio of Fe:OB were formed, which can be noticed from the slightly changed shape of the curve. Definitely, in further research, it is necessary to determine the mechanism of this reaction in order to confirm these effects. Stilbene OBs contain a double bond in their molecular structure, which can exist in cis and trans isomer. Exposure to sunlight can result in a decrease or loss of their fluorescence due to cis/trans isomerization [35], with the trans isomer fluorescing strongly while the cis isomer has no fluorescence. The absorption peaks of the cis isomer are much stronger. Their fluorescence emission peaks are in the range of 400-650 nm [36].

Since UV-C rays of the sunlight spectrum do not reach the Earth's surface [32-35], only UV-A and UV-B components of the sunlight spectrum are important for protective phenomena. The absorption and fluorescence spectra of biphenyl OB for three different concentration ranges are shown in Figure 3. The optical brightener biphenyl exhibits two absorption peaks, one in the UV-A region (at a wavelength of $347 \mathrm{~nm}$ ) and one in the UV-C region (at a wavelength of $246 \mathrm{~nm}$ ), Figure 3a. The peak of the fluorescence maximum of biphenyl OB is at $430 \mathrm{~nm}$ (Figure $3 \mathrm{~b}$ ).

Iron ions at the concentrations used did not affect the absorption characteristics of biphenyl OB in the UV-A region of the spectrum, confirming its stability in this region, (Figure 4a and Table 5). Despite the absorption stability of biphenyl OB being comparable to that of stilbene $\mathrm{OB}$, the iron ion caused a quench in the fluorescence of both derivatives, Figures $2 \mathrm{~b}$ and $4 \mathrm{~b}$.

\subsection{Whiteness Degree and Spectral $a^{*}$ and ${ }^{*} b^{*}$ Coordinates of Cotton Fabric}

In the treatment of cotton fabrics, the influence of iron ions on optical effects and UV protection effects was additionally investigated. The values of whiteness degree for cotton fabrics treated with single-bath treatment can be seen in Figures 5 and 6.

Whiteness degree increased with an increasing concentration of OBs, as expected. The influence of iron ions on the whiteness of cotton fabrics depends on OB derivative and treatments. Their influence in solutions of OBs is observed by fluorescence quenching (Figures $2 \mathrm{~b}$ and $4 \mathrm{~b}$ ). This phenomenon can be explained by the fact that iron ions cause fluorescence quenching, which is more pronounced in solutions than on a textile fabric. When treated with one bath, the differences in whiteness were less pronounced than when treated with two-bath treatment. Obviously, metal ions in a single-bath procedure created complexes with the molecules of stilbene optical brightener and reduced its fluorescence potential. A dramatic decrease of the whiteness of S_0.25 at $0.5 \mathrm{mg} / \mathrm{L}$ Fe and then increase at $1 \mathrm{mg} / \mathrm{L} \mathrm{Fe}$ is specific to the two-bath treatment. The reason for the decrease is a specific and stable interaction of $0.5 \mathrm{mg} / \mathrm{L} \mathrm{Fe}$ with cotton fabric. The concentration of $1 \mathrm{mg} / \mathrm{L} \mathrm{Fe}$ caused less effect because it was adsorbed and desorbed through movement in a Linitest apparatus. Biphenyl OB is more stable according to the proposed evaluation criteria.

The effect of iron ions added to the fluorescent compounds on washed cotton fabrics was monitored using the CIEL*a* $\mathrm{b}^{*}$ color space lightness in Table 6 and $\mathrm{a}^{*} \mathrm{~b}^{*}$ coordinates, Figure 7.

The addition of iron ions to OBs in single- and two-bath treatments did not affect the lightness of cotton fabrics (Table 6). Optical brighteners applied at higher concentrations, in interacting with iron ions, altered the $\mathrm{a}^{*}$ and $\mathrm{b}^{*}$ values. The changes were more pronounced in two-bath treatment. The trend was more evident with stilbene $\mathrm{OB}$ at the concentration of $0.25 \% w / w$ and $0.5 \mathrm{mg} / \mathrm{L}$ iron ions (Figure 7).

Iron ions influenced the negative value of $b^{*}$ coordinate of treated cotton fabric. This phenomenon can be explained by the formation of complex between iron ions and $\mathrm{OB}$ stilbene type, since the newly formed complex had less pronounced fluorescent properties, visible as a decrease in fluorescence intensity (Figure $2 b$ ). The effect was more pronounced with lower concentrations of OB. Furthermore, the iron ion solution was slightly paleyellow-colored, which is obviously sufficient to alter the fabric hue. Due to the present yellowish hue of the iron solution, it strongly affected the $-\mathrm{b}^{*}$ coordinate at lower OBs con- 
centrations and in two-bath treatment. Namely, the a two-bath treatment, the cotton fabric was treated with iron ions first, followed by washing in a detergent solution containing OB. In this case, the iron ions were bound on cotton fabric, so the washing process with a low amount of OBs was not sufficient to neutralize the fabric chromacity. It was a somewhat different situation in the case of the single-bath treatment. The impact was less pronounced in a single bath due to the formation of a complex with a different ratio of Fe:OB, and quenching occurred.

\subsection{UPF}

The equation for calculating the UPF clearly shows that the UV-C part of the spectrum (radiation of the wavelengths below $280 \mathrm{~nm}$ ) is not included, since this radiation does not reach the earth's surface. The absorption results (Tables 4 and 5) for the OBs solutions show that biphenyl and stilbene derivative absorb in the UV-A and UV-C. The addition of iron ions changes the absorption spectra of stilbene OB. The absorption maximum of UV-C was shifted to higher wavelengths, to UV-B, Table 4.

Untreated cotton fabrics exhibited a negligible degree of protection (UPF 7.276), mainly due to their structural properties and prebleaching processing level [7]. According to the influence of iron ions on stilbene $\mathrm{OB}$ in solution, expressed by the complex form, it was expected that the UPF of treated cotton fabrics would be increased. The UPF results of cotton fabric after single- and two-bath treatments were only slightly increased despite differences in absorption and fluorescence. The highest UV protection (UPF 15.923) was observed on cotton fabric after single-bath treatment with stilbene OB at $0.25 \%$ concentration with the highest concentration of iron ions $(1.0 \mathrm{mg} / \mathrm{L})$. Two-bath treatment, especially at $0.25 \% w / w$, reduced the UPF, which may be attributed to the ion-exchange properties of cotton cellulose and its reaction with iron ions (Figures 8 and 9).

The impact of iron ions on UPF at single- or two-bath treatments is not easy to analyze unambiguously. The results presented for the change of shade in cotton fabrics treated with fluorescent compounds with the presence of metal ions can also have an impact on the UV protection properties, as fabric chromacity (presence of a color, Figure 7) results in higher UPF values. In contrast to stilbene $\mathrm{OB}$, two-bath treatment of cotton fabric with biphenyl $\mathrm{OB}$ at higher concentrations of iron ions slightly reduced the UPF value. The equation used to calculate UPF clearly shows that the UV-C part of the spectrum (radiation of the wavelengths below $280 \mathrm{~nm}$ ) is not included, as this radiation does not reach the Earth surface. Absorption results (Table 5) for the florescent compound solutions show that biphenyl OB absorbs in this part of the spectrum as well. This fact is directly reflected in the lower UPF values of the fabric treated with a biphenyl derivative.

\section{Conclusions}

The influence of iron ions on optical brighteners in a solution and on treated cotton fabric was studied by analyzing of optical and protective phenomena. Two parameters, absorption and fluorescence spectra of optical brighteners in a solution, were compared with three parameters on cotton fabric: whiteness degree, hue changes, and UPF.

The results obtained showed that iron ions with stilbene as optical brightener produce a different species that absorbs in the UV-B region compared to the parent compound. It can be assumed that it is a new complex form between the stilbene optical brighteners and the iron ions.

In contrast, in the case of the biphenyl optical brightener, the iron ions had no effect on the absorption characteristics in the UV-A part of the spectrum, confirming its stability in this region. However, the influence of iron ions is noticeable by an increased absorption of the biphenyl optical brightener in the UV-C region.

The addition of iron ions in solution reduced the fluorescence intensity of the optical brighteners stilbene and biphenyl at all applied concentrations. The effect of iron ions on the absorption spectra of stilbene optical brightener in the form of bathochromic shift to 
the UV-C region, and on the fluorescence spectra of stilbene and biphenyl derivatives in the forming of fluorescence, quenching was observed.

The values of whiteness degree for cotton fabrics treated with a fluorescent compound with the addition of iron ions are generally lower in the case of the single-bath treatment. It has been proved that the metal ions form complex with the molecules of the optical brightener stilbene during one bath treatment, thereby reducing its potential.

The obtained results proved that the absorption and fluorescence as optical behavior in the solutions cannot be fully transferred to the optical and protective properties of the cotton fabrics obtained in single- and two-bath treatments with the detergent containing optical brighteners. We suggest that the contribution of the detergent partly caused a dispersive composition in which the influence of iron ions was suppressed.

Author Contributions: Conceptualization, T.P., T.D. and I.S.; methodology, T.D., B.V. and J.V.V.; formal analysis, T.D. and B.V.; investigation, T.D.; data curation, B.V. and J.V.V.; writing-original draft preparation, T.D. and T.P.; writing-review and editing, T.P., I.S., B.V. and J.V.V.; supervision, T.P. and I.S. All authors have read and agreed to the published version of the manuscript.

Funding: This work was partly supported by Project Mobility of Higher Education Professors at the University of Maribor (UM) 2018-2021, Contract No.: C3330-19-953001, co-funded by the European Union-European Social Fund and the Ministry of Education, Science and Sport.

Institutional Review Board Statement: Not applicable.

Informed Consent Statement: Not applicable.

Data Availability Statement: Data available in a publicly accessible repository.

Conflicts of Interest: The authors declare no conflict of interest.

\section{References}

1. Mustalish, R.A. Optical brighteners: History and technology. Stud. Conserv. 2000, 45 (Suppl. 1), 133-136. [CrossRef]

2. Skoog, D.A.; Holler, F.J.; Crouch, S.R.; Skoog, D.A. Principles of Instrumental Analysis, 6th ed.; Douglas, A., Skoog, F., James, H., Stanley, R.C., Eds.; Brooks/Cole Publishing Co.: Pacific Grove, CA, USA, 2007.

3. Shore, J. Fluorescent brightening agent. In Colorants and Auxiliaries: Organic Chemistry and Application Properties, 2nd ed.; Shore, J., Ed.; Society of Dyers and Colourists: Bradford, UK, 2002; Volume 2, pp. 760-812.

4. Tiki, A.; Amin, A.; Kanwal, A. Chemistry of optical brighteners and uses in textile industries. Pak. Text. J. 2010, $59,42-43$.

5. Bayly, A.E.; Smith, D.J.; Roberts, N.S.; York, D.W.; Capeci, S. Detergent Processing. In Handbook of Detergents, Part F: Production; Zoller, U., Sosis, P., Eds.; CRC Press by Taylor \& Francis Group: New York, NY, USA, 2009; pp. 323-363.

6. Kostajnšek, K.; Dimitrovski, K. Use of Extended Cover Factor Theory in UV Protection of Woven Fabric. Polymers 2021, $13,1188$. [CrossRef]

7. Zimniewska, M.; Batog, J. Ultraviolet-blocking properties of natural fibres. In Handbook of Natural Fibres; Kozlowski, R.M., Ed.; Woodhead Publishing Ltd.: Cambridge, UK, 2012; Volume 2, pp. 141-164.

8. Hoffmann, K.; Kaspar, K.; Gambichler, T.; Altmeyer, P. In vitro and in vivo determination of the UV protection factor for lightweight cotton and viscose summer fabrics: A preliminary study. J. Am. Acad. Dermatol. 2000, 43, 1009-1016. [CrossRef] [PubMed]

9. Zhu, J.; Li, H.; Wang, Y.; Wang, Y.; Yan, J. Preparation of Ag NPs and Its Multifunctional Finishing for Cotton Fabric. Polymers 2021, 13, 1338. [CrossRef]

10. Urbas, R.; Kostajnšek, K.; Dimitrovski, K. Impact of structure and yarn colour on UV properties and air permeability of multilayer cotton woven fabrics. Text. Res. J. 2011, 81, 1916-1925. [CrossRef]

11. Dobnik Dubrovski, P.; Golob, D. Effects of Woven Fabric Construction and Color on Ultraviolet Protection. Text. Res. J. 2009, 79, 351-359. [CrossRef]

12. Stanković, S.B.; Popović, D.; Poparić, G.B.; Bizjak, M. Ultraviolet Protection Factor of Gray-state Plain Cotton Knitted Fabrics. Text. Res. J. 2009, 79, 1034-1042. [CrossRef]

13. Achwal, W.B. UV Protection by Textiles. Colourage 2000, 44, 50-51.

14. Pezelj, E.; Tomljenović, A.; Čunko, R. Textiles for the Protection against Sun Radiation. Tekstil 2004, 53, 301-316.

15. Cox Crews, P.; Katchman, S.; Beyer, A. Influences on UVR Transmission of Undyed Woven Fabrics. Text. Chem. Colorists 1999, 31, 17-26.

16. Kim, J.; Stone, J.; Crews, P.; Shelley, M., II; Hatch, K.L. Improving Knit Fabric UPF Using Consumer Laundry Products: A Comparison of Results Using Two Instruments. Fam. Consum. Sci. Res. J. 2004, 33, 141-158. [CrossRef]

17. Bajaj, P.; Kothari, V.K.; Ghosh, S.B. Some innovations in UV protective clothing. Indian J. Fibres Text. Res. 2000, 35, 315-329. 
18. Eckhardt, C.; Rohwer, H. UV protector for cotton fabrics. Text. Chem. Colorist Am. Dyest. Report. 2000, 32, 21-23.

19. Heinemann, G.W.; Merkle, G. Determination of Optical Brighteners in Laundry Detergents. In Handbook of Detergents, Part C, Analysis; Waldhoff, H., Spilker, R., Eds.; Marcel Dekker, Inc.: New York, NY, USA, 2005; pp. 487-506.

20. Zappone, M.; Kaziska, A.; Bogush, G. Applications of detergent in laundering. In Handbook of Detergents, Part E: Applications; Zoller, U., Ed.; CRC Press by Taylor \& Francis Group: New York, NY, USA, 2009; pp. 69-82.

21. Salas, H.; Gutiérrez-Bouzán, C.; López-Grimau, V.; Vilaseca, M. Respirometric Study of Optical Brighteners in Textile Wastewater. Materials 2019, 12, 785. [CrossRef]

22. Saravanan, D. UV protection textile materials. AUTEX Res. J. 2007, 7, 53-62.

23. Stanford, D.G.; Georgouras, K.E.; Pailthorpe, M.T. Sun protection afforded by a summer weight garment: Effect of wash and wear. Med. J. Aust. 1995, 8, 422-425. [CrossRef] [PubMed]

24. Stanford, D.G.; Georgouras, K.E.; Pailthorpe, M.T. The effect of laundering on the sun protection afforded by a summer weight garment. J. Eur. Acad. Dermatol. Venereol. 1995, 5, 28-30. [CrossRef]

25. Gies, P. Photoprotection by clothing, Photodermatology. Photoimmunol. Photomed. 2007, 23, 264-274. [CrossRef]

26. Sarkar, A.K. On the relationship between fabric processing and ultraviolet radiation transmission. Photodermatol. Photoimmunol. Photomed. 2007, 23, 191-196. [CrossRef]

27. Soljačić, I.; Čunko, R. Wirkung von Kupfer-und Eisensalzen auf die Weißeffekte optisch aufgehellter Baumwolle. Melliand Text. 1979, 60, 1032-1038.

28. Milligan, B.; Holt, L. Fluorescent whitening agents I Bis-4,4'-(4"-methoxy-6"-phenoxy-s-triazin-2"'-ylamino)stilbene-2,2'disulphonic acid: Its photodecomposition in solution and on wool. Aust. J. Chem. 1974, 27, 195-203. [CrossRef]

29. Blanco, M.; Jiménez, L.; Valverde, I. Stability of a Stilbene-Type Fluorescent Whitening Agent against Hypochlorite. Text. Res. J. 2001, 71, 130-134. [CrossRef]

30. Mainali, B.; Pham, T.T.; Ngo, H.H.; Guo, W. Maximum allowable values of the heavy metals in recycled water for household laundry. Sci. Total Environ. 2013, 452, 427-432. [CrossRef] [PubMed]

31. Available online: https://www.farbechtheit.info/pdfs/sicherheitsdatenblaetter/DEK_ECE77_E.PDF (accessed on 14 August 2021).

32. EN ISO 105-J02:2000: Textiles-Tests for Colour Fastness_Part J02: Instrumental Assessment of Relative Whiteness; BSI: London, UK, 2000.

33. AS/NZS 4399:1966 Standard Test Method for Sun Protective Clothing Evaluation and Classification. In Standards Australian: Homebush, Australia; Standards New Zealand: Wellington, New Zealand, 1996.

34. Chitichotpanya, P.; Chitichotpanya, C. In Vitro Assessment of Sericin-Silver Functionalized Silk Fabrics for Enhanced UV Protection and Antibacterial Properties Using Experimental Design. Coatings 2017, 7, 145. [CrossRef]

35. Neiditch, O.W. Minor additives in heavy-duty laundry detergents. J. Am. Oil Chem. Soc. Vol. 1981, 58, 162-165. [CrossRef]

36. Grabchev, I. Photochemistry of some polymerizable fluorescent brighteners. J. Photochem. Photobiol. A Chem. 2000, 135, 41-44. [CrossRef] 